Bull. austral. Math. Soc.

$14 \mathrm{H} 20,13 \mathrm{H} 15$

Vol. $42(1990) \quad[1-6]$

\title{
ON THE SEMIGROUP OF AN ORDINARY MULTIPLE POINT
}

\author{
Devadatta M. Kulkarni
}

\begin{abstract}
In this paper we define the semigroup of an ordinary multiple point of an analytic plane curve $f$. This semigroup on tuples of integers is completely characterised in terms of the order of $f$, that is the number of distinct tangent directions to $f$ at that point.
\end{abstract}

In this paper we define the semigroup of an ordinary multiple point of an analytic plane curve $f$ and characterise it completely in terms of the order of $f$ at this point, that is the number of distinct tangent directions to $f$ at this point. This is a step towards the study of semigroups of analytically reducible curves. The study of the semigroup of an analytically irreducible curve can be found in Abhyankar [1] and Angermüller [2]. In both these papers one may find connections between the generators of the semigroup of an analytically irreducible curve, the characteristic pairs associated with it and its multiplicity sequence. The study of semigroups on tuples of integers corresponding to analytically reducible plane curves having two branches can be found in Garcia [4] and Bayer [3]. The general results about semigroups of analytically reducible curves are not known.

In Section 1 we define the semigroup of an ordinary multiple point of an analytic plane curve $f$. In Section 2 we give its characterisation in the Theorem.

\section{Notation and Terminology}

Let $A=k[[X, Y]]$ be a formal power series ring in two indeterminates $X$ and $Y$ with coefficients in an algebraically closed field $k$. For any $f(X, Y)$ in $A$, by $\operatorname{ord}_{A} f(X, Y)$ we denote the order of $f$ in $X$ and $Y$ and by $\operatorname{ord}_{T} g\left(T^{n}, \mathcal{N}(T)\right)$ we denote the order of $g\left(T^{n}, \mathcal{N}(T)\right)$ in $k[[T]]$ after a substitution $X=T^{n}$ and $Y=\mathcal{N}(T)$ in any $g(X, Y)$ in $A$ where $T$ is an indeterminate over $k$ and $\mathcal{N}(T) \in k[[T]]$ with $\mathcal{N}(0)=0$.

\section{Received 27 July 1989}

I would like to express my gratitude towards Professor Abhyankar for his continuous encouragement. I would like to thank Purdue University for its support and the Bhaskaracharya Pratishthana, Pune, India for its generous hospitality.

Copyright Clearance Centre, Inc. Serial-fee code: 0004-9729/90 \$A2.00+0.00. 
Let $f(X, Y) \in A$ with ord $A(X, Y)=d>0$, be written as

$$
f(X, Y)=f_{d}(X, Y)+f_{d+1}(X, Y)+\ldots
$$

where $f_{d}(X, Y) \not \equiv 0$. Except for at most a homogeneous linear change in $X$ and $Y$, we may assume that $f_{d}(0, Y) \neq 0$. If we substitute $X=X^{\prime}$ and $Y=X^{\prime} Y^{\prime}$ in $f_{d}(X, Y)$, we get.

$$
f_{d}\left(X^{\prime}, X^{\prime} Y^{\prime}\right)=X^{\prime d} f_{d}\left(1, Y^{\prime}\right)=c X^{\prime d}\left[\prod_{i=1}^{d}\left(Y^{\prime}-\alpha_{i}\right)\right]
$$

where $0 \neq c \in k$ and for $\alpha_{i} \in k, 1 \leqslant i \leqslant d$. $f$ is said to have an ordinary multiple point at the origin if and only if $\alpha_{i} \neq \alpha_{j}$ for $i \neq j, 1 \leqslant i, j \leqslant d$. By a known result, for such $f$ in $A$ we can write

$$
f(X, Y)=c f^{(1)}(X, Y) f^{(2)}(X, Y) \ldots f^{(d)}(X, Y)
$$

where for $1 \leqslant i \leqslant d, f^{(i)}(X, Y) \in A$ and where each $f^{(i)}(X, Y)$ looks like $f^{(i)}(X, Y)=$ $Y-\alpha_{i} X+$ higher degree terms in $X$ and $Y$. By the Weierstrass Preparation Theorem, for $1 \leqslant i \leqslant d$, we have

$$
f^{(i)}(X, Y)=U^{(i)}(X, Y)\left[Y-\mathcal{N}_{i}(X)\right]
$$

where $U^{(i)}(X, Y)$ is a unit in $A$ and $\mathcal{N}_{i}(X) \in k[[X]]$ where $\mathcal{N}_{i}(X)=\alpha_{i} X+$ higher degree terms in $X$. Thus $f(X, Y)$ can be written as

$$
f(X, Y)=U(X, Y) \prod_{i=1}^{d}\left[Y-\mathcal{N}_{i}(X)\right]
$$

where $U(X, Y)$ is a product of $c$ and $U^{(i)}(X, Y) 1 \leqslant i \leqslant d$. We define the $d$-tuple of integers

$$
\nu[f](g)=\left(\operatorname{ord}_{X} g\left(X, \mathcal{N}_{1}(X)\right), \operatorname{ord}_{X} g\left(X, \mathcal{N}_{2}(X)\right), \ldots, \operatorname{ord}_{X} g\left(X, \mathcal{N}_{d}(X)\right)\right)
$$

to be the branch multiplicity of $f$ with any $g$ in $A$. We may note that the definition of the branch multiplicity of $f$ with any $g$ does not depend upon an expression $\left(^{*}\right)$ of $f$. For $f$ as above, we set

$$
S=\left\{\nu[f](g): g(X, Y) \in A \text { and } g\left(X, \mathcal{N}_{i}(X)\right) \neq 0 \text { for } 1 \leqslant i \leqslant d\right\} .
$$

We observe that $S$ forms a semigroup on $d$-tuples of nonnegative integers by componentwise addition because for $g(X, Y)$ and $h(X, Y)$ in $A$, we have

$$
\operatorname{ord}_{X} g\left(X, \mathcal{N}_{i}(X)\right)+\operatorname{ord}_{X} h\left(X, \mathcal{N}_{i}(X)\right)=\operatorname{ord}_{X}\left[g\left(X, \mathcal{N}_{i}(X)\right) h\left(X, \mathcal{N}_{i}(X)\right)\right]
$$

for $1 \leqslant i \leqslant d$. We call $S$ the branch semigroup associated with an ordinary multiple point of $f$ and denote it by Brsem(f). 


\section{Characterisation of the Branch semigroup}

We are going to use the same $f$ and Brsem(f) as described in Section 1.

Lemma 1. For any $g(X, Y)$ in $A$ of order $e$, if $g_{e}(X, \alpha X) \neq 0$ then $\operatorname{ord}_{X} g(X, \alpha X)=\operatorname{ord}_{A} g=\operatorname{ord}_{X} g(X, \mathcal{N}(X))$ where $\mathcal{N}(X)=\alpha X+$ higher degree terms in $X$.

Proof: For $e=0$, the proof is clear. For $e>0$, let

$$
g_{e}(X, Y)=\prod_{i=1}^{e}\left[\gamma_{i} X+\delta_{i} Y\right]
$$

for some $\gamma_{i}, \delta_{i} \in k$ for $1 \leqslant i \leqslant e$. With the substitution, we get

$$
g(X, \mathcal{N}(X))=\prod_{i=1}^{e}\left[\gamma_{i} X+\delta_{i} \mathcal{N}(X)\right]+g_{e+1}(X, \mathcal{N}(X))+\ldots
$$

which becomes

$$
g(X, \mathcal{N}(X))=\prod_{i=1}^{e}\left[\gamma_{i}+\delta_{i} \alpha\right] X^{e}+\text { higher degree terms in } X .
$$

As $g_{e}(X, \alpha X) \neq 0$, we have $\left[\prod_{i=1}^{e}\left(\gamma_{i}+\delta_{i} \alpha\right)\right] \neq 0$ and so $\operatorname{ord}_{X} g(X, \mathcal{N}(X))=e=$ $\operatorname{ord}_{A} g$. Thus it is clear that

$$
\operatorname{ord}_{X} g(X, \alpha X)=\operatorname{ord}_{A} g==\operatorname{ord}_{X} g(X, \mathcal{N}(X)) .
$$

Lemma 2. Let $\left(e_{1}, \ldots, e_{d}\right) \in B r s e m(f)$ and $m=\min \left\{e_{i}: 1 \leqslant i \leqslant d\right\}$. Let $g(X, Y) \in A$ such that $\operatorname{ord}_{X} g\left(X, \mathcal{N}_{i}(X)\right)=e_{i}$ for $1 \leqslant i \leqslant d$. If $m<d$ then $\operatorname{ord}_{A} g=m$.

PROOF: Let $\operatorname{ord}_{A} g=m^{\prime}$. If $m^{\prime}=0$ it is clear that $m=m^{\prime}=\operatorname{ord}_{A} g$. We note that $m \geqslant \operatorname{ord}_{A} g$ since $e_{i}=\operatorname{ord}_{X} g\left(X, \mathcal{N}_{i}(X)\right) \geqslant \operatorname{ord}_{A} g=m^{\prime}$ for $1 \leqslant i \leqslant d$. Suppose $0<m^{\prime}<m$. If we write

$$
g_{m^{\prime}}(X, Y)=\prod_{i=1}^{m^{\prime}}\left(\gamma_{i} X+\delta_{i} Y\right)
$$

for some $\gamma_{i}, \delta_{i} \in k$ for $1 \leqslant i \leqslant m^{\prime}$, we note that at most $m^{\prime}$ of $\left\{Y-\alpha_{i} X: 1<i \leqslant d\right\}$ divide $g_{m^{\prime}}(X, Y)$. As $m^{\prime}<d$, we have at least one $t, 1 \leqslant t \leqslant d$ such that $Y-\alpha_{t} X$ does not divide $g_{m^{\prime}}(X, Y)$. By Lemma 1 , we have

$$
e_{t}=\operatorname{ord}_{X} g\left(X, \mathcal{N}_{t}(X)\right)=\operatorname{ord}_{X} g\left(X, \alpha_{t} X\right)=m^{\prime} .
$$


This contradicts the definition of $m$. So $m^{\prime}=m$ that is $\operatorname{ord}_{A} g=m$.

TheоREM. Given a positive integer $d$, let $\left(e_{1}, e_{2}, \ldots, e_{d}\right)$ be a $d$-tuple of nonnegative integers. Let $m=\min \left\{e_{i}: 1 \leqslant i \leqslant d\right\}$. Then

$$
\left(e_{1}, e_{2}, \ldots, e_{d}\right) \in B r s e m(f)
$$

if and only if

$$
m \geqslant d \text { or } \operatorname{card}\left(\left\{i: e_{i}=m\right\}\right) \geqslant d-m \text {. }
$$

Proof: Part I: Assume that $\left(e_{1}, e_{2}, \ldots, e_{d}\right) \in \operatorname{Brsem}(f)$. Let $m=\min \left\{e_{i}: 1 \leqslant\right.$ $i \leqslant d\}$. Suppose $m<d$. There exists $g(X, Y) \in A$ such that ord $\operatorname{or}_{X} g\left(X, \mathcal{N}_{i}(X)\right)=e_{i}$ for $1 \leqslant i \leqslant d$. By Lemma 2 , ord $\operatorname{lom}_{A}=m$. Let

$$
g_{m}(X, Y)=\prod_{i=1}^{m}\left(\gamma_{i} X+\delta_{i} Y\right)
$$

where $\gamma_{i}, \delta_{i} \in k$ for $1 \leqslant i \leqslant m$. At most $m$ of $\left\{Y-\alpha_{i} X: 1 \leqslant i \leqslant d\right\}$ divide $g_{m}(X, Y)$; and by Lemma 1 , for each $t$ where $Y-\alpha_{t} X$ does not divide $g_{m}(X, Y)$, we have

$$
\operatorname{ord}_{X} g\left(X, \mathcal{N}_{t}(X)\right)=e_{t}=\operatorname{ord}_{A} g=m \text {. }
$$

Thus $\operatorname{card}\left(\left\{i: e_{i}=m\right\}\right) \geqslant d-m$.

Part II: Let $\left(e_{1}, e_{2}, \ldots, e_{d}\right)$ be a $d$-tuple of nonnegative integers. Let $m=$ $\min \left\{e_{i}: 1 \leqslant i \leqslant d\right\}$.

ClaIM. If $m \geqslant d$ then

$$
\Phi(X, Y)=\sum_{i=1}^{d}\left\{\prod_{\substack{j \neq i \\ 1 \leqslant j \leqslant d}}\left(Y-\mathcal{N}_{j}(X)\right)\right\} X^{e_{i}-d+1}
$$

is in $A$ such that

$$
\operatorname{ord}_{X} \Phi\left(X, \mathcal{N}_{i}(X)\right)=e_{i} \quad \text { for } 1 \leqslant i \leqslant d .
$$

Proof of THE ClaIM. We note that for each $i, e_{i}-d+1>0$ as $e_{i} \geqslant m \geqslant d$. For each $i$

$$
\begin{aligned}
\Phi\left(X, \mathcal{N}_{i}(X)\right) & =\left[\prod_{\substack{i \neq j \\
1 \leqslant j \leqslant d}}\left(\mathcal{N}_{i}(X)-\mathcal{N}_{j}(X)\right)\right] X^{e_{i}-d+1} \\
& =\left[\prod_{\substack{i \neq j \\
1 \leqslant j \leqslant d}}\left(\alpha_{i}-\alpha_{j}\right) X^{d-1}+\text { terms in } X \text { of degree }>d-1\right] X^{e_{i}-d+1} \\
& =\left\{\prod_{\substack{i \neq j \\
1 \leqslant j \leqslant d}}\left(\alpha_{i}-\alpha_{j}\right)\right\} X^{e_{i}}+\text { terms in } X \text { of degree }>e_{i} .
\end{aligned}
$$


Thus $\operatorname{ord}_{X} \Phi\left(X, \mathcal{N}_{i}(X)\right)=e_{i}$ for $1 \leqslant i \leqslant d$.

Claim. Let $m<d$ and $\operatorname{card}\left(\left\{i: e_{i}=m\right\}\right)=d-u$ where $u \leqslant m$. Without loss of generality we may assume that

$$
e_{1}=e_{2}=e_{3}=\cdots=e_{d-u}=m \text {. }
$$

We have

$$
\begin{aligned}
\Psi(X, Y)= & {\left[\prod_{d-u+1 \leqslant j \leqslant d}\left(Y-\mathcal{N}_{j}(X)\right)\right] X^{m-u} } \\
& +\sum_{i=d-u+1}^{d}\left\{\prod_{\substack{j \neq i \\
d-u+1 \leqslant j \leqslant d}}\left(Y-\mathcal{N}_{j}(X)\right)\right\} X^{e_{i}-u+1}
\end{aligned}
$$

in $A$ such that

$$
\operatorname{ord}_{X} \Psi\left(X, \mathcal{N}_{i}(X)\right)=e_{i} \quad \text { for } 1 \leqslant i \leqslant d
$$

Proof of The claim. For $d-u+1 \leqslant i \leqslant d$, we note that $m-u \geqslant 0$ and $e_{i}-u+1>0$ since $e_{i}>m \geqslant u$. For $1 \leqslant k \leqslant d-u$, we have

$$
\begin{aligned}
\Psi\left(X, \mathcal{N}_{k}(X)\right)= & \left\{\prod_{d-u+1 \leqslant j \leqslant d}\left(\mathcal{N}_{k}(X)-\mathcal{N}_{j}(X)\right)\right\} X^{m-u} \\
& +\sum_{i=d-u+1}^{d}\left\{\prod_{\substack{j \neq i \\
d-u+1 \leqslant j \leqslant d}}\left(\mathcal{N}_{k}(X)-\mathcal{N}_{j}(X)\right)\right\} X^{e_{i}-u+1} \\
= & {\left[\left\{\prod_{d-u+1 \leqslant j \leqslant d}\left(\alpha_{k}-\alpha_{j}\right)\right\} X^{m}+\text { terms in } X \text { of degree }>m\right] } \\
& \left.+\sum_{i=d-u+1}^{d}\left[\int_{\substack{j \neq i \\
d-u+1 \leqslant k \leqslant d}}\left(\alpha_{k}-\alpha_{j}\right)\right\} X^{e_{i}}+\text { terms in } X \text { of degree }>e_{i}\right]
\end{aligned}
$$

Noting that $e_{i}>m$ for $d-u+1 \leqslant i \leqslant d$, we have $\operatorname{ord}_{X} \Psi\left(X, \mathcal{N}_{k}(X)\right)=m=e_{k}$ for 
$1 \leqslant k \leqslant d-u$. For $d-u+1 \leqslant k \leqslant d$, we have

$$
\begin{aligned}
\Psi\left(X, \mathcal{N}_{k}(X)\right) & =\left\{\prod_{\substack{j \neq k \\
d-u+1 \leqslant j \leqslant d}}\left(\mathcal{N}_{k}(X)-\mathcal{N}_{j}(X)\right)\right\} X^{e_{k}-u+1} \\
& =\left\{\prod_{\substack{j \neq k \\
d-u+1 \leqslant j \leqslant d}}\left(\alpha_{k}-\alpha_{j}\right)\right\} X^{e_{k}}+\text { terms in } X \text { of degree }>e_{k} .
\end{aligned}
$$

Thus we have $\operatorname{ord}_{X} \Psi\left(X, \mathcal{N}_{k}(X)\right)=e_{k}$ for $d-u+1 \leqslant k \leqslant d$.

Remark about The PRoOf of THE THEOREM. In the claims in Part II, we can also take respectively

and

$$
\Phi(X, Y)=\sum_{i=1}^{d}\left\{\prod_{\substack{j \neq i \\ 1 \leqslant j \leqslant d}}\left(Y-\alpha_{j} X\right)\right\} X^{e_{i}-d+1}
$$

$$
\begin{aligned}
\Psi(X, Y)= & \left\{\prod_{d-u+1 \leqslant j \leqslant d}\left(Y-\alpha_{j} X\right)\right\} X^{m-u} \\
& +\sum_{i=d-u+1}^{d}\left\{\prod_{\substack{j \neq i \\
d-u+1 \leqslant j \leqslant d}}\left(Y-\alpha_{j} X\right)\right\} X^{e_{i}-u+1}
\end{aligned}
$$

which are members of $k[X, Y]$ satisfying the requirements.

\section{REFERENCES}

[1] S. Abhyankar, 'On the semigroup of meromorphic curve', (Part I), in International Symposium on Algebraic Geometry, Kyoto, pp. 249-414, 1977.

[2] G. Angermüller, 'Die Werthalbgruppe einer Ebenen Irreduziblen Algebroiden Kurve', Math. Z. 153 (1977), 267-282.

[3] V. Bayer, 'Semigroup of two irreducible algebroid plane curves', Manuscripta Math. 49 (1985), 207-241.

[4] A. Garcia, 'Semigroups associated to singular points of plane curves', J. Reine Angew. Math. 336 (1982), 165-185.

Current address

Department of Mathematics

University of Poona

Pune 411007

India
Department of Mathematical Sciences

Oakland University

Rochester MI 48063

United States of America 